Revista Calle14, Volumen 8 Número 12 / enero - abril de 2014, ISSN 2011-3757
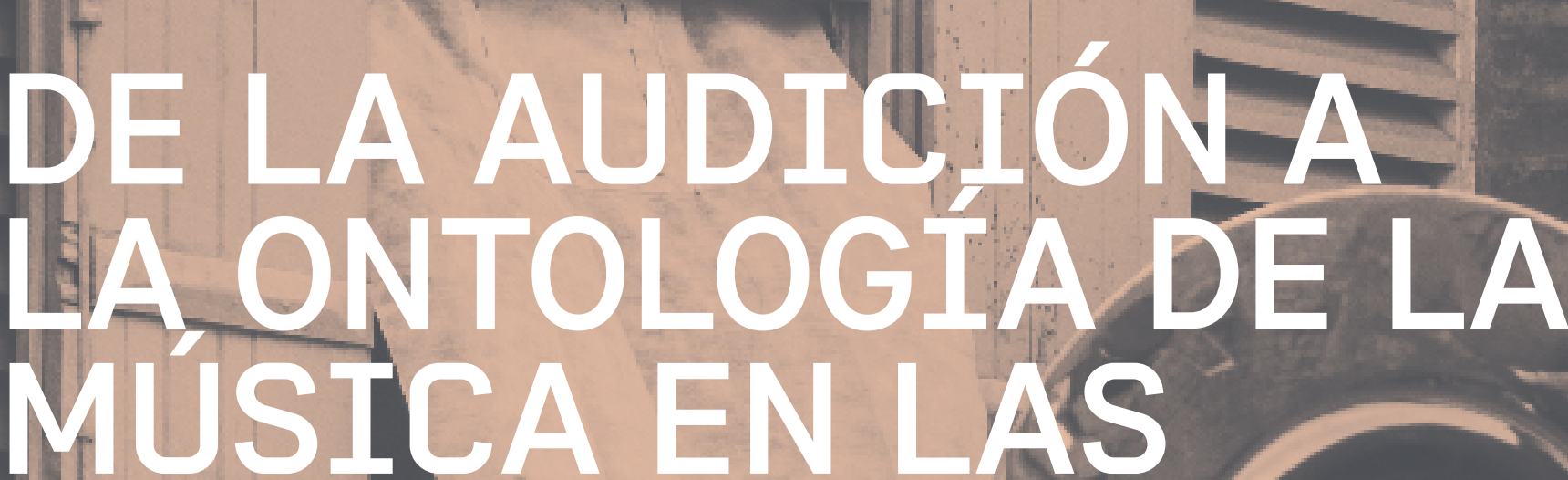

ASIGNATURAS DE MÚSICA Y CONTEXTO

DEL PROYECTO CURRICULAR

DE ARTES MUSICALES, UDFJC

Artículo de investigación

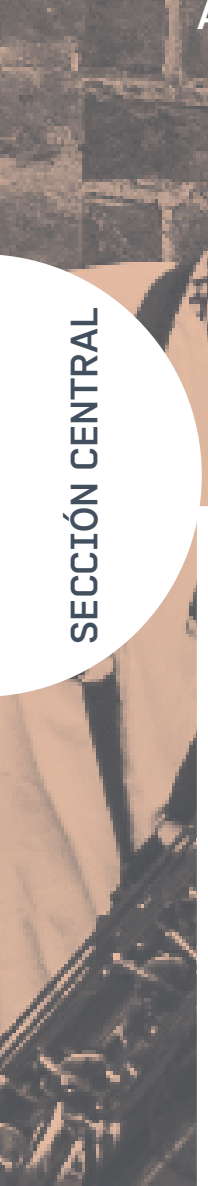

\title{
Francisco Castillo
}

Universidad Distrital Francisco José de Caldas / franciscanisimo@hotmail.com

\section{Genoveva Salazar}

Universidad Distrital Francisco José de Caldas / salazar_genoveva@yahoo.com

\section{Pilar Agudelo}

Universidad Distrital Francisco José de Caldas / mapiaguval@gmail.com

\section{Manuel Bernal}

Universidad Distrital Francisco José de Caldas / bandolo_bernal@yahoo.com

El equipo de investigación está conformado por Francisco Castillo, intérprete de música barroca y docente en Historia y análisis; Manuel Bernal, intérprete, arreglista y docente en el campo de músicas tradicionales;

Pilar Agudelo y Genoveva Salazar, investigadoras y docentes en el campo de la audición musical. Como equipo trabajan desde el año 2010 en el proyecto de investigación "La audición musical: concepciones, propuestas y prácticas de desarrollo en el Proyecto Curricular de Artes Musicales de La Universidad Distrital Francisco José De Caldas", dirigido por Genoveva Salazar Hakim. Algunos de los resultados parciales de este trabajo han sido presentados en el X Encuentro de la Sociedad Argentina para las Ciencias Cognitivas de la Música (SACCOM), en la II Jornada de la Escuela de Música de la Universidad el Rosario, Argentina; en el VI encuentro del Foro Latinoamericano de Educación Musical y en el Encuentro de Investigaciones de la ASAB. 
CATILLO F, SALAZAR G, AGUDELO P, BERNAL M (2014) De la audición a la ontología de la música en las asignaturas de música y contexto del proyecto curricular de artes musicales, UDFJC.

Calle14,8 (12), 66-79.

\title{
RESUMEN
}

El ejercicio pedagógico de asociar las prácticas musicales con el entorno diverso de los actores involucrados conduce a usar formas concretas de entender la naturaleza tanto del conocimiento musical como de las maneras en que dicho conocimiento es adquirido. ¿Qué es concebido como práctica de audición en las asignaturas que propenden por esta contextualización? ¿Cómo inciden estas prácticas de audición en la concepción de música y conocimiento musical? El objetivo de este texto es indagar por las ontologías de la música presentes en las asignaturas del área de Música y Contexto que se derivan de su relación con las prácticas de audición musical en el aula. Se analizan cualitativamente las respuestas que dieron los docentes de esta área a una entrevista estructurada, identificando ahí tendencias generales y particularidades en materia de ontologías de la música y formas de conocimiento musical.

\section{PALABRAS CLAVES}

Audición musical, ontología de la música, contexto, conocimiento musical

\section{FROM MUSICAL HEARING TO THE ONTOLOGY OF MUSIC IN THE COURSES OF MUSIC AND CONTEXT OF THE MUSICAL ARTS CURRICULUM AT UDFJC}

\begin{abstract}
The pedagogical exercise of associating musical practices with the varied backgrounds of the agents involved leads to specific ways of understanding the nature of both musical knowledge and the paths through which such knowledge is acquired. What is understood as the practice of musical hearing in the courses that promote this contextualization? How do these practices affect hearing in the conception of music and musical knowledge? The purpose of this paper is to explore the ontologies of music present in the courses of the area of Music and Context derived from its relationship to musical hearing practices in the classroom. The answers given by teachers to a structured interview were qualitatively analyzed, identifying general trends and particularities surrounding the ontologies of music and musical forms of knowledge.
\end{abstract}

\section{KEYWORDS}

Musical hearing, ontology of music, context, musical knowledge

\section{DE L'AUDITION À L'ONTOLOGIE DE LA MUSIQQUE DANS LES THÈMES DE MUSIQQUE ET CONTEXTE DU PROJET PÉDAGOGIQUE DES ARTS MUSICAUX, UDFJC}

\section{RÉSUMÉ}

L'exercice pédagogique d'associer les pratiques musicales à l'univers différent des acteurs impliqués conduit à utiliser des moyens concrets de comprendre la nature tant de la connaissance 
musicale que des façons dont cette connaissance est acquise. Qu'entend-on en tant que pratique d'audition dans les thèmes qui tendent vers cette contextualisation? Comment ces pratiques d'audition affectent la conception de la musique et la connaissance musicale? Le but de cet article est d'étudier les ontologies de la musique présentes dans les thèmes dans le domaine de la Musique et du Contexte dérivés de sa relation avec les pratiques d'écoute de la musique dans l'auditoire. Les réponses données par les enseignants dans ce domaine lors d'une entrevue structurée sont analysées qualitativement, identifiant ainsi des tendances générales et des particularités en matière d'ontologies de la musique et des formes de connaissances musicales.

\section{MOTS CLÉS}

Audition musicale, ontologie de la musique, contexte, connaissance musicale

\section{DA AUDIÇÃO À ONTOLOGIA DA MÚSICA NAS MATÉRIAS DE MÚSICA E CONTEXTO DO PROJETO CURRICULAR DE ARTES MUSICAIS, UDFJC}

\section{RESUMO}

O exercício pedagógico de associar as práticas musicais com o entorno diverso dos atores envolvidos conduz a usar formas concretas de entender a natureza tanto do conhecimento musical como das maneiras em que dito conhecimento é adquirido. 0 que é concebido como prática de audição nas matérias que propendem por esta contextualização? Como incidem estas práticas de audição na concepção da música e do conhecimento musical? O objetivo deste texto é indagar pelas ontologias da música presentes nas matérias da área de Música e Contexto que se derivam de seu relacionamento com as práticas de audição musical na aula. Analisam-se qualitativamente as respostas que deram os docentes desta área numa entrevista estruturada, identificando tendências gerais e particulares em matéria de ontologias da música e formas de conhecimento musical.

PALAVRAS-CHAVE:

Audição musical, ontologia da música, contexto, conhecimento musical.

\section{TUKUIKUNATA UIACHISKAKUNATA UIASPA IACHAIKUIKUNAPI IACHAIKUITA APACHINGAPA TAKIIKUNA IACHANGAPA,UDFJC}

\section{SUGLLAPI MAILLA KILKAILLATA}

Iachaikuskata sugllapi takiikunata takispa. Iacharimi ima imasapi uiachinkuna sugkunamanda, ¿imatak imasatak uiachingui iachaikunata munankuna kaikunawa Sugllapi? ¿ imasatak kai iachaikunata uiaspa iachari takiikuna iachagta? Kaiwa munarikumi, iachanga tukuima takiikuna takiipa Iachaikuikunapi chasak allilla uiaspa Iachaikukungapa. Iachaikudiru ukupi iacharimi iuiarispa ainirkakuna, iachachigkuna kai Iachachiipi. Rimanakuspa kawaspa tukuipi takiikunapi imasami iachaikuri.

\section{IMA SUTI RIMAI SIMI}

Takiita uiai, tukui takiikuna, takiikunata rigsig, suglla kilkai. 


\section{Introducción}

En febrero del año 2010, un grupo de profesores y estudiantes del Proyecto Curricular de Artes Musicales (PCAM) dieron inicio a un proyecto de investigación que tenía como propósito visibilizar distintos aspectos de las prácticas de audición musical en diversas asignaturas de la malla curricular. Dicho proyecto, titulado "La audición musical: concepciones, propuestas y prácticas de desarrollo en el Proyecto Curricular de Artes Musicales de La Universidad Distrital Francisco José De Caldas", contaba con la participación de Genoveva Salazar como investigadora principal y Manuel Bernal, Pilar Agudelo y Francisco Castillo como investigadores auxiliares. El presente artículo hace parte del mencionado proyecto de investigación, procurando enfocarse en un conjunto concreto de asignaturas pertenecientes al área de Música y Contexto.

\section{La investigación}

Si bien el proyecto se apoyaba en iniciativas de investigación previas dentro del área (Salazar 2011, 2007 y 2005), este proyecto tenía como detonante la diversidad de las tradiciones musicales que integran nuestro plan de estudios, tal y como se ve en el documento "Solicitud de Registro Calificado de Programas Académicos de Educación Superior":

El planteamiento es similar al de otros programas en cuanto que se rige por los cánones de la educación musical superior en los circuitos nacionales e internacionales, lo que se refleja en elementos tan variados como la titulación, las denominaciones y contenidos generales de muchas asignaturas, la adopción de cierta periodización histórica, el uso de metodologías que provienen de comunidades académicas de larga tradición, el estudio de instrumentos musicales y repertorios propios de los desarrollos históricos de las culturas centrales a nivel mundial, entre otros aspectos. La diferencia radica en que el plan de estudios ofrece una combinación única en la que los estudiantes entran en contacto con las prácticas musicales académicas ya citadas y además con las prácticas musicales locales, regionales y populares de tradición oral y/o escrita. ${ }^{1}$

Siendo nuestro campo el lugar de la audición musical en un contexto que se plantea la educación musical desde distintas tradiciones, las problemáticas generadas se expresan en interrogantes como ¿Qué es concebido como audición musical en los espacios académicos? ¿Qué propuestas en materia de audición musical están expresadas en los programas (syllabus)? ¿Qué tipo de prácticas de audición podemos observar en las clases?

La observación de un fenómeno tan complejo como la audición ha merecido una revisión detallada de las metodologías, modelos de investigación y un marco conceptual que permita interpretar la información recogida.

Para esto último, la búsqueda de un marco conceptual, los integrantes del proyecto establecimos un seminario, en el que compartimos lecturas, ideas, procedimientos y conceptos pertinentes a nuestros intereses. Dicho seminario, desarrollado desde el 2010 hasta el 2012, contó con la participación de los profesores del proyecto y de estudiantes de música, para quienes el espacio ha servido de apoyo a sus trabajos de grado y sus intereses personales, a la vez que nutren la investigación institucional.

En comunión con las tradiciones musicales ajenas a la académica occidental, es de especial relevancia considerar las distintas maneras de conocer la música. Para esto resulta útil evocar los tres modos primarios de conocer

Documento "Solicitud de Registro Calificado de Programas Académicos de Educación Superior", p. 10, que se presentó ante el Ministerio de Educación y el Consejo Nacional de Acreditación en 2006 y que se constituye en el principal referente conceptual del programa. 

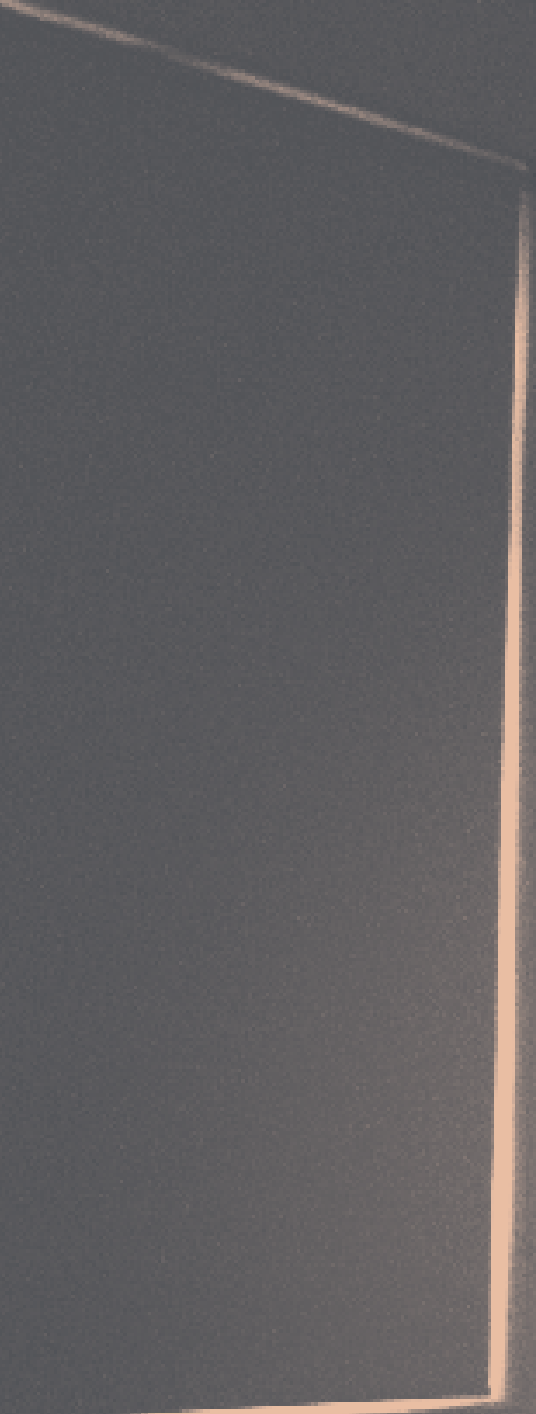
la música (Davidson y Scripp, 1992): la producción, la percepción y la reflexión. La producción, como modo de conocer la música, es el componente inicial y abarca una amplia gama de pensamiento musical: por ejemplo, cómo se expresa en la composición o en la ejecución interpretativa. La percepción sustenta la formación de sonidos físicamente presentes en la ejecución en vivo y en la audición silenciosa desde la partitura. Sin la percepción, hacer música sería inasible en sus elementos sensoriales. El reconocimiento del rol esencial de volver a imaginar, reconceptualizar y volver trabajar una composición musical o una ejecución interpretativa constituye el núcleo de la reflexión como modo de conocer.

Estas ideas se entrelazan con dos posibles condiciones del conocimiento (Davidson y Scripp, 1992): por fuera de la ejecución y dentro de la ejecución. La primera está relacionada con la forma declarativa o fija del conocimiento, y la segunda asociada a la forma procedimental o dinámica del mismo.

De la asociación de las dos condiciones del conocimiento con los tres posibles modos de conocer resultan seis situaciones específicas que ubican una experiencia musical en dos dimensiones simultáneas: la producción, la reflexión y la percepción dentro y fuera de la acción.

El concepto antes mencionado de conocimiento procedimental es susceptible de ser asociado a los fundamentos filosóficos que plantea Stubley (1992) como el modo de conocer, en paralelo no excluyente de el qué conocer, denominado también conocimiento proposicional.

Como parte de los mismos fundamentos, es mencionado el empirismo. Este enfoque posa su mirada sobre la percepción de elementos del sonido (timbre, altura, duración, intensidad) y posteriormente sobre cómo estos elementos son entendidos, lo cual da surgimiento a estudios sobre habilidades y capacidades para la música. Indaga también en las subdestrezas que subyacen a diversas prácticas musicales, en habilidades de escucha y respuesta a la música.

Nuestro uso de los fundamentos filosóficos de Stubley como marco de interpretación de los resultados finaliza con la validación de la composición, la interpretación y la audición como distintas maneras de acceder al conocimiento musical, cada una con características particulares pero afines.

En cuanto a la composición como modo de conocimiento, Stubley presenta el enfoque de la gramática generativa transformacional de la música, que se basa a su vez en el estructuralismo y arroja luces respecto de los principios sintácticos y los patrones musicales particulares dentro de ciertas tradiciones estilísticas. La variedad de destrezas técnicas que constituyen el acto de la ejecución musical son el cuerpo de la ejecución como modo de conocimiento, junto con el acto de hacer música en sí mismo, donde pensamiento y acción se integran. La audición como modo de conocimiento se identifica típicamente con la percepción estética, la cual se desarrolla a través de cinco niveles de respuesta al estímulo que aparecen de forma sucesiva: (1) La reacción esencialmente sensorial, (2) la respuesta centrada alrededor de los conceptos de representación y disciplina con el centro de atención en lo que despierta la obra de arte, (3) la validez de las propiedades afectivas de la obra, (4) el nivel de respuesta enfocado a los medios, la forma y el estilo y (5) la respuesta que cuestiona los criterios, conceptos y valores con los cuales una tradición construye los significados artísticos (Stubley, 1992). Este último nivel de respuesta al estímulo posee un vínculo directo con las iniciativas curriculares que dan forma al plan de estudios nuestro PCAM.

Estos distintos acercamientos al proceso de conocimiento musical dirigen el discurso de manera inevitable a una consideración del concepto de música en sí mismo. Diversas tradiciones musicales que confluyen en un solo programa académico son un escenario muy fértil a la caracterización e interrelación entre variadas concepciones de la música. Los datos que arroja nuestro estudio en esta materia son vistos a la luz de la definición de ontologías de la música que propusiera P. Bohlman (2001). El autor hace una descripción y clasificación, en buena medida sustentada en estudios etnomusicológicos, de numerosas concepciones-ontologías que, de la música, se tienen en situaciones variadas. Cabe resaltar ahí la aparente dicotomía que surge al concebir la música como objeto, o como proceso. En la ontología de la música como objeto, esta se constituye como un ente ajeno, aislado y delimitado, susceptible de ser estudiado. No es el mismo caso para la ontología como proceso, donde la música puede existir como actividad, de forma indefinida y de límites abiertos. De la misma forma, Bohlman (2001) alude a dos condiciones ontológicas de posible identificación en los procesos musicales, como lo son el adumbramiento, término prestado de las artes plásticas para explicar que la música no se nos presenta directamente, sino a través del efecto que produce, y el incrustacionismo, condición que sostiene que un estímulo musical se presenta 
acompañado de otras actividades. Esta perspectiva de interpretación se ve enriquecida por una mirada a la ontología de la música en la tripartición música como texto, música como interpretación y música como movimiento, elaborada en Vargas, López \& Shifres (2007).

Habida cuenta de la enorme magnitud del fenómeno que pretendemos observar, en este artículo nuestro interés concreto está en analizar lo que, en materia de audición musical, ocurre dentro de las asignaturas de música y contexto, y hacerlo desde una perspectiva ontológica y epistémica.

Dos espacios académicos conforman el área de música y contexto: Músicas Regionales y Sistemas Musicales.

Músicas Regionales tiene dos niveles que son cursados durante un año. En el desarrollo de la asignatura se toman elementos de dos áreas de conocimiento: la teoría musical y la historia social de la música. En lo pertinente a la teoría se trabajan herramientas orientadas al análisis musical para abordar las músicas locales-regionales colombianas en sus niveles constitutivos y en sus aspectos performativos, tanto desde la partitura como desde otras fuentes documentales sonoras y audiovisuales. Al abordar el contenido histórico la asignatura enmarca los contenidos musicales en el contexto socio-cultural del cual surgen, dando así relevancia a la función social de la música, a las características de su producción, circulación y consumo-apropiación, a la cultura en la cual actúan y al pensamiento que acompaña su práctica.

Sistemas Musicales tiene cinco niveles, en los cuales se distribuye el estudio de la historia de la música occidental desde la antigüedad hasta el presente. De forma muy similar a lo que ocurre en Músicas Regionales, Sistemas Musicales busca establecer vínculos que enlacen la historia de nuestra cultura con sus manifestaciones artísticas a través de la historiografía, la teoría musical y el análisis musical. Son estudiadas aquí las distintas épocas de la historia de la música, sus personajes, obras representativas, corrientes de pensamiento y ejercicios performativos desde distintas metodologías, que incluyen la lectoescritura, la composición, el análisis y la interpretación.

El instrumento de recolección de información elegido para el componente de la investigación presente en este artículo fue la Entrevista Estructurada, desarrollada a partir de siete preguntas abiertas, realizada por correo electrónico a todos los docentes del área de Música y Contexto (cinco profesores).

Las preguntas realizadas fueron:

1. Si su asignatura propicia el desarrollo de competencias auditivas en los estudiantes, indique cuáles considera que desarrolla.

2. Describa cuáles contenidos musicales teóricos, técnicos e interpretativos se articulan directamente con las prácticas de audición musical que tienen lugar en sus clases.

3. Indique si las prácticas de audición musical que realiza en su clase requieren de desarrollos previos de audición fundamentados en otros espacios de formación.

4. ¿Qué otro tipo de contenidos son relevantes en su asignatura y de qué manera se articulan con las prácticas de audición musical?

5. En caso de realizar análisis musical vinculando la audición musical, indique en qué autores, métodos, teorías o experiencias se basa su propuesta de análisis.

6. En el caso de utilizar guías o cuestionarios para realizar actividades de análisis musical vinculando la audición, haga una descripción del tipo de contenidos que se indagan en dichos análisis.

7. Describa algunas de las situaciones de su clase en las que no encuentra pertinente realizar prácticas de audición musical.

En relación con las predicciones del proyecto, las clases de Música y Contexto incluyen una serie de prácticas de audición que permiten ser analizadas según nuestros objetivos, entre los cuales se encuentra una caracterización que clasifica dichas prácticas en tres categorías:

- $\quad$ Función: ¿Cuál es la función que cumplen en estos espacios las prácticas de audición? ¿Qué propósitos persiguen? Dentro de esta categoría, se reconocieron de antemano dos funciones generales como lo son la apropiación y la producción. De la misma forma cada una de estas subcategorías se ve dividida en unidades de análisis más específicas como la comprensión de aspectos musicales, la adquisición de modelos de referencia, el monitoreo de la ejecución o la generación de discurso o de competencias auditivas.

- Contenidos: ¿Qué contenidos se vinculan con las prácticas de audición? Similar al caso anterior, se divide en dos categorías intermedias que 
delimitan los contenidos conceptuales y los contenidos metodológicos, para luego caracterizar más específicamente hacia el tipo concreto de contenidos.

- Materiales: ¿En qué se apoyan estas prácticas de audición? ¿Qué tipo de materiales se asocian con la audición? Esta categoría está fragmentada en varias unidades, que pretenden enmarcar los materiales de apoyo vinculados a la audición según el sujeto que ejecuta la acción, el sujeto autor del material, la presencia o no de partitura, el lugar de la acción y la tradición musical a la que pertenece.

\section{Resultados y discusión}

La observación de los materiales con que los docentes apoyan las prácticas de audición en sus clases, según lo expresado en la encuesta, permite corroborar, por un lado, que la asignatura Sistemas Musicales hace uso de documentos propios a la tradición académica occidental mientras Músicas Regionales combina entre materiales de tradición campesina, regional y académicos occidentales. En este caso, la tendencia sugiere el uso de materiales de tradición regional y campesina de apoyo en las prácticas de audición, y un apoyo en materiales de tradición académica occidental para las actividades de análisis musical.

A la indagación sobre las competencias auditivas promovidas en las clases de Música y Contexto, le son propios resultados que sugieren una alta presencia de contenidos metodológicos en materia de análisis formal e interpretativo de las obras, al tiempo que se evocan frecuentemente los vínculos presentes entre las prácticas auditivas, los contenidos teóricos y los contenidos contextuales. La función de estas prácticas de audición, se ve encaminada a fortalecer procesos como la comprensión de la estructura y del estilo musical.

Según los resultados, las actividades de audición musical que se dan en estas asignaturas se apoyan en otros espacios de formación dentro del currículo de estudios. Más en particular, esta asociación se da en términos de las estrategias que, vinculadas a la audición, toman la forma de análisis formal, a la vez que referencian la transversalidad de elementos propios a la teoría musical occidental. En el mismo sentido, cuando se pregunta abiertamente a los docentes por otro tipo de contenidos presentes en sus clases que estén relacionados con la audición, de manera general afirman que las prácticas auditivas en ellas tienen como propósito la producción de un discurso verbal o escrito, es decir, hablar o escribir sobre aquello que se escucha como una actividad que integra distintas competencias y contenidos. En tales prácticas, y en plena consonancia con la naturaleza de las asignaturas observadas, la aparición de elementos y contenidos de tipo contextual es bastante significativa.

Dado que el foco de interés para este trabajo es el lugar de la audición en asignaturas de contexto, un componente resaltado por los docentes, es el análisis musical. Resulta evidente que, en las actividades de este orden, la audición musical se considera parte del ejercicio, en especial una audición cuya función sea apropiarse de aspectos teórico-musicales por medio de operaciones tales como analizar, comprender, internalizar, abstraer o identificar aspectos de la estructura musical. De forma que el ejercicio analítico que convencionalmente hace uso extensivo de la partitura como documento se ve complementado con audiciones, que no solo ilustran los resultados de dicho análisis, sino que también hacen parte del análisis en sí mismo, tal y como se ve en respuestas como esta, en relación a las competencias auditivas:

Audición relacional, es decir, escuchando las relaciones que se manifiestan entre los elementos que se analizan y también entre los roles que se cumplen en los formatos. (Resultados de la encuesta realizada. Fuente: archivo de insumos del proyecto de investigación)

En casos como el que ha sido citado, puede leerse una concepción de la audición musical como parte constitutiva del análisis musical. De la misma forma, puede leerse también como una integración de distintos modos simultáneos de conocer la música (Davidson y Scripp, 1992), en la medida en que se entienda como tal al ejercicio mismo de percibirla mientras es ejecutada (o reproducida), junto con el acto reflexivo, que se desarrolla tanto durante la audición, como por fuera de ella. Ya que la audición interna trasciende en forma de expresión verbal o escrita de las relaciones una vez ha finalizado la audición externa, resuenan con esto las ideas de empirismo en la medida en que este que separa dos procedimientos: por un lado los elementos de la música son percibidos y, por otros, son entendidos en un sistema de relaciones. 
Del caso anterior y en conjunto con los demás resultados es evidente que el lugar del análisis musical incluye actividades de audición y es también un modo de conocer la música.

¿Qué modo de conocimiento es el análisis musical? ¿A qué concepto de música está aludiendo?

Si bien algunos de los procedimientos propios al análisis musical corresponden a la condición ontológica de OBJETO, en la medida que la obra musical se hace objeto en la partitura, también interviene ahí la ontología PROCESO, por la relevancia que se le da a la audición en función de comprender rasgos estructurales de la música, habida cuenta de la identificación de estructuras como parte sustancial del análisis musical propiamente dicho. Otras ontologías pueden interactuar en este fenómeno, como lo son el incrustacionismo, ya que durante el análisis son consideradas otras dimensiones, en ocasiones ajenas a la teoría musical:

Competencia auditiva: Audición analítica musical en relación con lo contextual y con categorías interdisciplinarias.

Es un reto enorme para todos nosotros, que yo siento frecuentemente en los alumnos, y es la necesidad de relacionar la música con otros aspectos de la vida, es decir, en música y contexto en serio se siente la necesidad de eso, la necesidad de poder darle vida propia, cómo esa obra musical tiene relación con tal fenómeno de literatura, o con tal proceso de crisis económica.... (Resultados de la encuesta realizada. Fuente: Archivo de insumos del proyecto de investigación).

De esta manera, la ontología compuesta que, desde la audición, se observa en las actividades de análisis musical, deviene en formas multimodales de conocimiento. La identificación de aspectos musicales en la audición corresponde a un tipo de conocimiento no proposicional, que se hace proposicional en el momento en que se expresa.

Quizá otras miradas de este fenómeno, que involucren otras herramientas, como la observación de las clases y de los documentos institucionales, nos permita configurar de mejor manera el tránsito pendular que tiene una obra musical al interior de una asignatura y en relación con la audición; no obstante, algo de ese oscilamiento puede verse en las respuestas de los maestros. La obra, desde la partitura, se instala como objeto en dicha ontología y se conoce de manera proposicional mediante el uso de ejercicios perceptivos y reflexivos por fuera de la acción misma de la audición. Posteriormente la obra se despoja de su límite como objeto (no queriendo decir con esto que se hace subjetiva); se concibe como proceso en la audición, se conoce de ella de forma procedimental por medio de operaciones como la percepción y la reflexión dentro de la ejecución, para volver de nuevo a su ontología de objeto en la producción del discurso sobre la misma, o en la materialización de los resultados del análisis.

La producción escritural que es fruto del análisis de la audición musical (Resultados de la encuesta realizada. Fuente: Archivo de insumos del proyecto de investigación).

Nuestros resultados sugieren que la obra musical alterna entre distintas ontologías en relación con la forma en que se conoce y en lo que se espera conocer de la música a través de ella, aunque se percibe una tendencia a concluir los ejercicios de audición y análisis con actividades propias a la expresión oral o escrita. Esta tendencia podría ser entendida como parte de los hábitos propios del ejercicio docente, que implica emitir una calificación y para lo cual resulta más práctico calificar sobre un objeto palpable; pero según lo que señalan los maestros encuestados, la producción de discurso sobre la música no es llanamente un requerimiento académico: La reflexión por fuera de la ejecución se entiende como un modo de conocer la música, que si bien se asocia con conocimiento de tipo declarativo, hace uso de ejercicios reflexivos y perceptivos durante el momento mismo de la audición, es decir, que integra al conocimiento declarativo un componente no declarativo o procedimental.

En nuestra observación de las respuestas, surge una tendencia para apoyar la variedad que, en materia de ontologías, se presenta en los espacios. La presencia alta de la categoría "prácticas de audición vinculadas a contenidos metodológicos alusivos a herramientas de análisis formal" conlleva a asignarle a la obra una condición de objeto, segmentándolo, reagrupándolo y quizá ejerciendo un nominalismo en las unidades formales. Junto con esta categoría, es también significativa la presencia de audiciones que persiguen como propósito el conocimiento de aspectos estructurales así como audiciones articuladas a contenidos contextuales, semánticos y valorativos. Puestas en conjunto, se trabaja desde la partitura con una obra musical a manera de objeto, pero al mismo tiempo se relaciona 
consigo misma (estructura), con el entorno (contenidos contextuales) y con otras disciplinas o con dimensiones afectivas o valorativas de los estudiantes.

Una coincidencia con los presupuestos del proyecto se ve claramente en los tipos de materiales que son usado como apoyo a las prácticas auditivas: todos los espacios hacen uso de grabaciones y de repertorio acorde a la tradición musical que les concierne. No obstante, se pueden hacer visibles algunos desbalances, como lo es el uso frecuente de la partitura en asignaturas cuyo campo de estudio se apoya en un repertorio donde la tradición oral tiene altísima importancia. Otras miradas, apoyadas en herramientas investigativas complementarias a la entrevista darán nuevas luces sobre este caso.

En un aspecto relacionado, es frecuente encontrar materiales de la tradición académica occidental en las actividades de Músicas Regionales, usados como fundamentación para abordar su repertorio. Tal es el caso de enfoques y propuestas de análisis musical, que aunque aplicadas a músicas tradicionales, tienen su origen en la tradición occidental. No ocurre lo complementario en Sistemas Musicales, espacio académico enfocado en la historia de la música occidental en que no hay presencia de materiales de la tradición popular, regional o campesina. Para discusiones posteriores sobre el tema, urge mirar cómo los materiales de apoyo a la audición que se usan en Músicas Regionales pueden aportar a las audiciones de música de tradición académica, no solo en términos de articulación curricular, sino en aras de enriquecer los ejercicios de escucha analítica y sobre todo de contextualización de los eventos musicales, materia en la cual la música de tradición campesina y regional tiene mucho que aportar en materia de procedimientos analíticos

El trabajo que se proyecta y estimula desde este análisis implica dos líneas de trabajo. Por un lado es necesario cruzar la información de las entrevistas realizadas con otros insumos, como la observación de clases, los documentos institucionales y otras herramientas de naturaleza cuantitativa. Por otro lado, la fundamentación teórica puede ser enriquecida, en la perspectiva de fortalecer las categorías de análisis en nuestra investigación. Podrían ser muy útiles ahí los debates que en torno al concepto de obra musical se dan desde campos como la semiótica y la filosofía, así como es importante también vincular los resultados con estudios de cognición situada y corporeidad.

\section{Referencias}

Bohlman, Philip V. (2001). "Ontologies of Music", en Cook, N., \& Everist, M., (Eds.), Rethinking Music (pp. 17-34) Oxford: Oxford University Press.

Castillo, F; Salazar, G; Agudelo, P. y M. Bernal (2011). "Caracterización de las prácticas de audición musical en asignaturas de formación instrumental", en A. Guiena; P. Jaquier; M. Valles y M. Martínez (Eds.) Musicalidad Humana: Debates actuales en evolución, desarrollo, cognición e implicancias socio-culturales. Buenos Aires: SACCOM

Davidson, L. and Scripp, L. (1992). "Surveying the Coordinates of Cognitive Skills in Music", en R. Colwell (Eds.) Handbook of Research on Music Teaching and Learning. New York: Schirmer Books.

Proyecto Curricular de Artes Musicales (2006). Solicitud de Registro Calificado. Bogotá: Universidad Distrital Francisco José de Caldas. (Documento institucional)

Salazar, G. (2005). “La ubicación tonal en jóvenes que inician el proceso de lectura melódica tonal entonada", en Actas de las I Jornadas de Educación Auditiva. La Plata: Facultad de Bellas Artes, Universidad Nacional de La Plata.

Salazar, G. (2007). “La lectura musical: procesos perceptivos, motores y cognitivos y sus vínculos con las estrategias de agrupación de la información escrita", en Calle 14: Revista de investigación en el campo del arte. ISSN-e 2145-0706, №. 1, 2007

Salazar, y M.O. Piñeros (2007). “Estrategias para resolver dificultades de entonación en jóvenes que inician procesos de lectura y dictado melódico". (Informe de investigación). Bogotá: Universidad Distrital Francisco José de Caldas.

Salazar, G. y M.O. Piñeros (2008). "Taller de Pedagogía Vocal: propuestas de estrategias para resolver dificultades de entonación", en Calle 14: Revista de investigación en el campo del arte, ISSN-e 2145-0706, №. 2, 2008

Salazar, y M.O. Piñeros (2007). “Estrategias para resolver dificultades de entonación en jóvenes que inician procesos de lectura y dictado melódico". (Informe de 
investigación). Bogotá: Universidad Distrital Francisco José de Caldas.

Salazar, G; Castillo, F; Bernal, M. y P. Agudelo (2011) "Prácticas de audición en asignaturas teórico musicales y de contexto", en A. Guiena; P. Jaquier; M. Valles y M. Martínez (Eds.) Musicalidad Humana: Debates actuales en evolución, desarrollo, cognición e implicancias socio-culturales. Buenos Aires: SACCOM

Stubley, E. V. (1992). "Philosophical Foundations", en R. Colwell (Ed.). Handbook of Research in Music Teaching and Learning. Reston: MENC - Shirmer Books.

Vargas, G.; López, I.; Shifres, F. (2007). “Ontología de la música en la educación auditiva", en: M. Espejo (Ed.) Actas de la II Jornada de Educación Auditiva. Tunja: Facultad de Ciencias de la Educación, UPTC. 